\title{
Upregulation of phosphatase and tensin homolog is essential for the effect of 4-aminopyridine on A549/CDDP cells
}

\author{
ZHENGYI LUO ${ }^{1,2^{*}}$, JIAFENG WANG ${ }^{1,3^{*}}$, CHENGLIN LI ${ }^{1,4^{*}}$, YUMIAO QIU $^{1}$, JING HUANG $^{1,5}$, \\ YUJIE HUANG ${ }^{1}$, HONGLI GU ${ }^{1}$, BIN WU ${ }^{1}$, ZHE HU $^{1,6}$ and YAN ZHEN ${ }^{1}$ \\ ${ }^{1}$ Institute of Respiratory Diseases, Affiliated Hospital of Guangdong Medical University, Zhanjiang, \\ Guangdong 524001; ${ }^{2}$ Department of Neurobiology, Southern Medical University, Guangzhou, \\ Guangdong 510515; ${ }^{3}$ Stem Cell Research and Clinical Translation Center, Affiliated Hospital of \\ Guangdong Medical University, Zhanjiang, Guangdong 524001; ${ }^{4}$ Department of Oncology, \\ Linyi People's Hospital, Linyi, Shandong 276000; ${ }^{5}$ Oncology Center; ${ }^{6}$ Department of Anesthesiology, \\ Affiliated Hospital of Guangdong Medical University, Zhanjiang, Guangdong 524001, P.R. China
}

Received September 21, 2017; Accepted January 29, 2018

DOI: $10.3892 / \mathrm{mmr} .2018 .8585$

\begin{abstract}
AP), a voltage-gated potassium channel blocker, was revealed to possess pro-apoptotic properties in various types of cancer cells. The present study aimed to explore the effect of 4-AP on a cisplatin (DDP) resistant lung cancer cell line A549/CDDP and the underlying mechanism by which it had an effect. In the present study, an MTT assay and cell cycle analysis were used to determine that 4-AP inhibited cell growth in vitro and a tumorigenesis assay in nude mice determined that 4-AP also inhibited cell growth in vivo. 4-AP induced cell apoptosis of A549/CDDP cells observed by electron microscopy and Annexin V-APC/7-ADD analysis. In addition, 4-AP enhanced the sensitivity of A549/CDDP cells to DDP as revealed by an MTT assay. Mechanistically, 4-AP upregulated the phosphatase and tensin homolog (PTEN) and modulated the phosphoinositide 3-kinase/protein kinase B signaling pathway and its downstream cell cycle factors, including cyclin D1, cyclin-dependent kinase 4 and p21, as well as apoptosis-associated proteins B-cell lymphoma 2, pro-caspase 9, pro-caspase 3, cleaved caspase 9 and cleaved caspase 3 . The effects of 4-AP on cell growth and apoptosis were reversed by PTEN silencing. In conclusion, the results indicated that 4-AP inhibited cell growth,
\end{abstract}

Correspondence to: Dr Yan Zhen or Dr Zhe Hu, Institute of Respiratory Diseases, Affiliated Hospital of Guangdong Medical University, 57 Renmin Road South, Zhanjiang, Guangdong 524001, P.R. China

E-mail: yingshuang288@163.com

E-mail: 379814602@qq.com

"Contributed equally

Key words: 4-aminopyridine, A549/CDDP, cell growth, cell apoptosis, cisplatin sensitivity, phosphatase and tensin homolog induced apoptosis and sensitized A549/CDDP cells to DDP via the upregulation of PTEN. 4-AP may be a potential therapeutic agent for patients with DDP resistance.

\section{Introduction}

Lung cancer is the most common cancer in the world, of which $80 \%$ were non-small cell lung cancer (NSCLC). Surgical resection is known as the most effective treatment for NSCLC, however, due to the fact that most diagnoses were confirmed in an advanced stage because of its deep location and no specificity of symptoms in its early stage, only a few patients can be cured by surgical treatment. Thus cisplatin (DDP) based adjuvant chemotherapy was studied as a standard treatment for patients with completely resected NSCLC (1). However, chemotherapy resistance usually occurs mainly due to DDP resistance, which contributes to a poor long-term survival rate of $15 \%$ (2). As one of the important mechanisms of drug resistance is resistant to DDP-induced cell apoptosis in lung cancer (3), therefore, finding an effective medicine to induce apoptosis of DDP resistance cells is a reasonable strategy to reverse resistance.

Accumulating evidence has indicated that 4-aminopyridine (4-AP), one of the most commonly used $\mathrm{K}^{+}$channel inhibitors, suppresses proliferation and induces apoptosis in various types of cancer cells, such as malignant astrocytoma (4), hepatoblastoma (5), acute myeloid leukemia and glioma $(6,7)$. Therefore, 4 -AP is presented as potential therapeutic agents for various types of cancers.

It has been shown that transmembrane current and activity of $\mathrm{K}^{+}$channels is significantly high in NSCLC (8). However, whether 4-AP could affect cell growth of A549/CDDP is not clear. In this study, we examined the effect and possible molecular basis of 4-AP in A549/CDDP cells and found that 4-AP inhibited cell growth, induced cell apoptosis and sensitized A549/CDDP cells to DDP via upregulating phosphatase and tensin homolog (PTEN). Together these results provide a novel mechanism for 4-AP as a potential therapeutic agent for patients with DDP resistance. 


\section{Materials and methods}

Cell culture. DDP resistant lung cancer cell line A549/CDDP was obtained from Oncology Center of our hospital and maintained in DMEM medium supplemented with $10 \%$ fatal bovine serum (FBS; PAA Laboratories; GE Healthcare, Chicago, IL, USA), and was incubated in a humidified chamber with $5 \% \mathrm{CO}_{2}$ at $37^{\circ} \mathrm{C}$.

Treatment of A549/CDDP cells with 4-AP. 4-AP was bought from Sigma-Aldrich; Merck KGaA, Darmstadt, Germany. Twenty-four hours prior to transfection, A549/CDDP cells were plated onto a 6-well plate or a 96-well plate (Nest Biotechnology Co., Ltd., Jiangsu, China) at 30-50\% confluence. Cells were treated with $7 \mathrm{mM}$ 4-AP and collected after $48 \mathrm{~h}$ for further experiments.

Cell viability. Cells were treated with different concentrations of 4-AP $(0,1.56,3.125,6.25$ and $12.5 \mathrm{mM})$ for $48 \mathrm{~h}$ or $7 \mathrm{mM}$ 4-AP for 1, 2 and 3 days. Cell viability was determined by MTT assay, as previously described (9). Experiments were performed three times.

Cell cycle analysis. Total of $5 \times 10^{6}$ cells were collected following treatment with $7 \mathrm{mM} \mathrm{4-AP}$ for $48 \mathrm{~h}$. Cell cycle analysis was performed according to the previous description (9). Each experiment was performed in triplicate.

In vivo tumorigenesis assay in nude mice. The treated groups were subcutaneously injected to the left flank of 4-6-week-old 12-13 g male BALB/c nu/nu mice $(\mathrm{N}=5)$ with a suspension of $8 \times 10^{6}$ A549/CDDP cells with 4-AP (7 mM). The control group were injected to the right flank of nude mice with a suspension of $8 \times 10^{6}$ A549/CDDP cells with 0.01 M PBS $(7,10)$. Tumor size was monitored using a calliper in the process of tumor growth and measured every 3 days. Mice were sacrificed using cervical dislocation 18 days after subcutaneous injection and tumor tissues were excised and weighed. Tumor volumes were calculated as follows: $\left(\mathrm{Dxd}^{2}\right) / 2$, where $\mathrm{D}$ is the longest diameter and $d$ is the shortest diameter. All animal studies were conducted in accordance with the principles and procedures outlined in Guangdong Medical University Guide for the Care and Use of Animals (Guangdong) 2011-020. All experiments procedures were approved by the The Animal Care and Use Committee of the Guangdong Medical University (no. GDY1701068).

Transmission electron microscopy. A549/CDDP cells were cultured in $10 \mathrm{~cm}$-diameter plates with $7 \mathrm{mM} 4$-AP treatment for 48 h. $5 \times 10^{6}$ cells were collected by centrifugation at 2,000 rpm for $10 \mathrm{~min}$ and washed twice with PBS. The pelleted cells were fixed in $2.5 \%$ cold glutaraldehyde supplemented with $0.1 \mathrm{M}$ of sodium cacodylate/1\% sucrose buffer for $24 \mathrm{~h}$. The cells were washed three times with PBS, then postfixed in $1 \%$ osmium tetroxide (60 min), encapsulated in $1 \%$ agar, stained with uranyl acetate and phosphotungstic acid, and dehydrated in a series of graded ethanolic solutions. Propylene oxide was added before the cells were finally embedded in Epon 812-Araldite mixture. Ultrathin sections $(50 \mathrm{~nm})$ were cut using ultramicrotome, placed under 200 mesh standard copper grids and examined under JEM-1400 transmission
Table I. Small interfering RNA sequences of PTEN.

\begin{tabular}{cl}
\hline Gene & Sequence \\
\hline PTEN & \\
1 & Sense: 5'-GAGCGUGCAGAUAAUGACAdTdT-3' \\
& Antisense: 3'-dTdTCUCGCACGUCUAUUACUGU-5' \\
2 & Sense: 5'-GUAUAGAGCGUGCAGAUAAdTdT-3' \\
& Antisense: 3'-dTdTCAUAUCUCGCACGUCUAUU-5' \\
3 & Sense: 5'-GUUAAAGAAUCAUCUGGAUdTdT-3' \\
& Antisense: 3'-dTdTCAAGGGCUUAGUAGACCUA-5'
\end{tabular}

PTEN, phosphatase and tensin homolog.

electron microscope. Each experiment was performed in triplicate.

Apoptosis assays. A549/CDDP cells were treated with $7 \mathrm{mM}$ 4-AP for $48 \mathrm{~h}$. Apoptosis was demonstrated by Annexin V-APC/7-ADD Apoptosis detection kit KGA1025 (Kaiji, Nanjing, China). Briefly, $1-5 \times 10^{5}$ cells were collected, washed twice in cold PBS, and resuspended in $500 \mu 1$ binding buffer. The suspension cells were stained with $5 \mu$ l Annexin V-APC and $5 \mu \mathrm{l}$ 7-ADD, and incubated for $15 \mathrm{~min}$ at room temperature in the dark. Apoptotic cells were assessed using FACS (BD Biosciences, Franklin Lakes, NJ, USA). Experiments were performed at least three times to qualify apoptosis by phosphatidylserine (PS) externalization.

Treatment of A549/CDDP cells with 4-AP and DDP. A549/CDDP cells were treated with different concentrations of 4-AP (3, 6 and $10 \mathrm{mM})$ and $\operatorname{DDP}(0,12.5,25,50,100$ and $200 \mu \mathrm{M}$ ) (Qilu Pharmo Co. Ltd, China) for $48 \mathrm{~h}$ at $37^{\circ} \mathrm{C}$. Cell viability was determined using MTT assay, as previously described (9). Experiments were performed three times.

Treatment of A549/CDDP cells with 4-AP and PTEN siRNAs. SiRNA for PTEN was designed and synthesized by Guangzhou RiboBio Co., Ltd., (Guangzhou, China) (Table I). The sequences of each gene and their controls are shown in Wang's study (11). A549/CDDP cells were treated with 4-AP (7 mM) and PTEN siRNAs (100 $\mathrm{nM})$ together for $48 \mathrm{~h}$ and collected for further experiments. Each experiments were performed three times.

Western blot analysis. Cells were lysed in RIPA buffer (Kaiji, Nanjing, China), and protein concentration was determined using BCA assay (Beyotime Institute of Biotechnology, Haimen, China). Total protein $(30 \mu \mathrm{g})$ was resolved using a $10 \%$ SDS-polyacrylamide gel electrophoresis (PAGE) gel and electro-transferred to polyvinylidene fluoride membranes (Invitrogen; Thermo Fisher Scientific, Inc., Waltham, MA, USA). Membranes were blocked with 5\% non-fat dry milk (for Phosphorylation antibody, adding BSA) in Tris-buffered saline ( $\mathrm{pH} 7.5$ ) with $0.1 \%$ Tween-20, followed by immunobloting overnight at $4^{\circ} \mathrm{C}$ with the following primary antibodies: Anti-pPI3K (Tyr458) (cat no. 4228S, 1:1,000), PI3K (cat no. 4249S, 1:1,000), pAkt (Ser473) (cat no. 4060S, 1:1,000), 
A

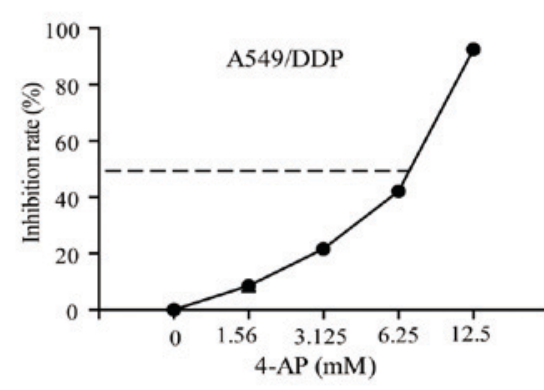

B
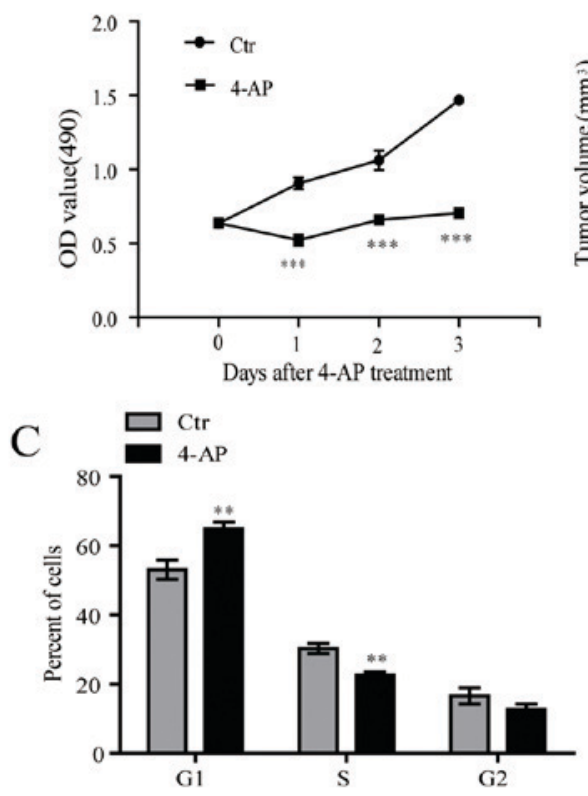
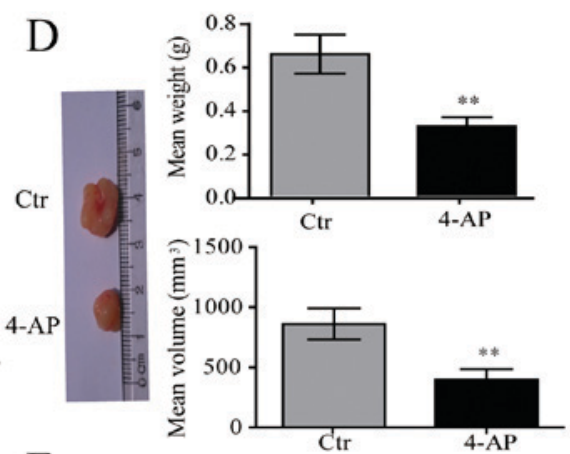

$\mathrm{E}$

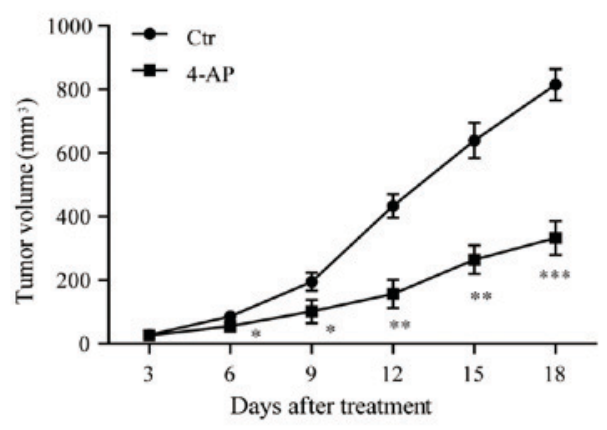

$\mathrm{F}$

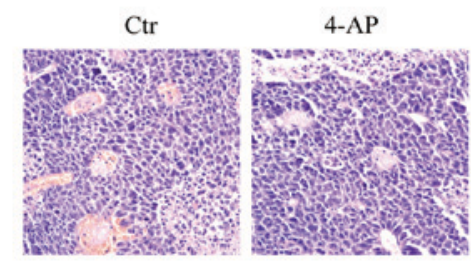

Figure 1. 4-AP inhibits cell growth in vitro and in vivo. (A) 4-AP suppressed cell viability in a dose-dependent manner in A549/CDDP cells. (B) 4-AP inhibited cell viability in A549/CDDP cells. (C) 4-AP blocked cell cycle transition from $\mathrm{G}_{1}$ to $\mathrm{S}$ and $\mathrm{G}_{2}$ phase in A549/CDDP cells. (D) The in vivo effectiveness of 4-AP was evaluated in xenograft mouse models bearing tumors originating from A549/CDDP cells. (E) Tumor volume was periodically tested for each mouse and tumor growth curve was plotted. (F) Hematoxylin-eosin staining of tumor tissues originated from the mouse models. Original magnification, x200; scale bar, $25 \mu \mathrm{m}$. Data were presented as mean \pm standard deviation for three independent experiments $\left({ }^{*} \mathrm{P}<0.05,{ }^{* *} \mathrm{P}<0.01,{ }^{* * * *} \mathrm{P}<0.001 \mathrm{vs}\right.$. the control). $4-\mathrm{AP}$, 4-aminopyridine; $\mathrm{Ctr}$, control.

Akt (cat no. 4691S, 1:1,000), CCND1 (cat no. 2978, 1:1,000), CDK4 (cat no. 12790, 1:1,000) and p21 antibody (cat no. 2947, 1:1,000) were all purchased from Cell Signaling Technology, Inc., (Danvers, MA, USA). Anti-PTEN (cat no. ab31392, 1:1,000), Bcl2 (cat no. ab32124, 1:1,000), pro-caspase 9 (cat no. ab135544, 1:1,000) and pro-caspase 3 (cat no. ab32150, 1:1,000), cleaved caspase 9 (cat no. ab2324, 1:1,000) and cleaved caspase 3 (cat no. ab2302, 1:1,000) were purchased from Abcam (Cambridge, UK). Anti- $\beta$-actin (cat no. 14395-1-AP, 1:1,000) was purchased from Proteintech (Rosemont, Illinois, USA). An HRP-conjugated anti-rabbit (cat no. SA00001-2, 1:1,000) or anti-mouse IgG antibody (cat no. SA00002-1, 1:1,000) purchased from ProteinTech Group Inc., (Chicago, IL, USA) was used as the secondary antibody for $1 \mathrm{~h}$ at room temperature. Signals were detected using enhanced chemiluminescence reagents (Pierce; Thermo Fisher Scientific, Inc.). Bands were analyzed using Image $\mathrm{J}$ and protein expression quantities were determined according to the following calculation: Integrated optical density (IOD)=density (mean) $\mathrm{x}$ area.
Statistical analysis. All data were analyzed for statistical significance using SPSS 13.0 software. Two-tailed Student's $t$ test was used for comparisons of two independent groups. One-way ANOVA was used to determine differences between groups for all in vitro analyses followed by S-N-K multiple comparison test. Repeated measures data of ANOVA was used to determine differences between groups in in vivo tumorigenesis assay. $\mathrm{P}<0.05$ was considered to indicate a statistically significant difference.

\section{Results}

4-AP inhibits cell growth in vitro and in vivo. DDP-resistant lung cancer cell line A549/CDDP, was treated with incremental doses up to $12.5 \mathrm{mM}$ of 4 -AP for $48 \mathrm{~h}$. 4-AP suppressed cell viability of A549/CDDP in a dose-dependent manner. The IC50 of 4-AP was $7 \mathrm{mM}$ (Fig. 1A), which was chosen for further experiments. The growth curves showed that 4-AP significantly inhibited cell growth of A549/CDDP 


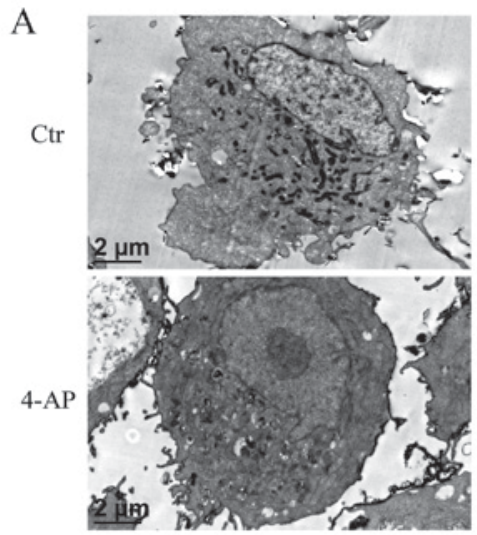

B

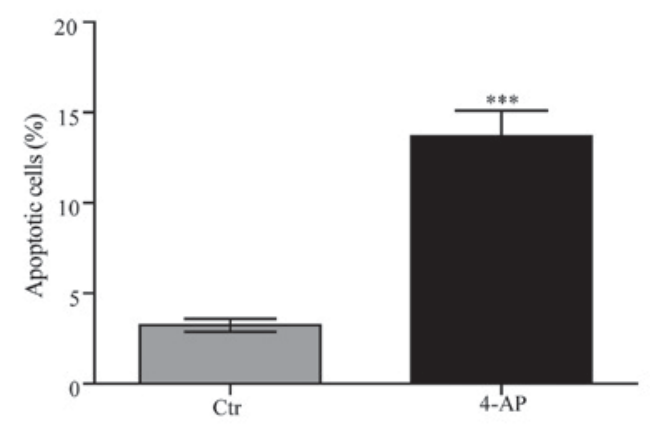

Figure 2. 4-AP induces cell apoptosis of A549/CDDP cells. (A) Nuclear chromatin condensation and apoptotic cells with 4-AP treatment for $48 \mathrm{~h}$ observed by electron microscopy. Original magnification, $\mathrm{x} 12,000$; scale bar, $2 \mu \mathrm{m}$. (B) Apoptotic cells with 4-AP treatment for $48 \mathrm{~h}$ detected by flow cytometry after Annexin V-APC/7-ADD double staining. Data were presented as mean \pm standard deviation for three independent experiments $\left({ }^{* * *} \mathrm{P}<0.001 \mathrm{vs}\right.$. the control). 4-AP, 4-aminopyridine; Ctr, control.

cells (Fig. 1B). Further, we observed that 4-AP blocked cell cycle transition from $\mathrm{G} 1$ to $\mathrm{S}$ and $\mathrm{G} 2$ phase in A549/CDDP cells (Fig. 1C). Subsequently, cell proliferation was measured in vivo by innoculating A549/CDDP cells into nude mice. The treated groups were subcutaneously injected to the left flank of nude mice with a suspension of $8 \times 10^{6}$ A549/CDDP cells with 4-AP (7 mM). The control group were injected to the right flank of nude mice with A549/CDDP cells suspension with normal saline (NS). Tumor volume was periodically tested once every other day until 18 days and growth curve was plotted. We observed that 4-AP obviously inhibited tumor growth compared with control (Fig. 1D-F). These above results suggest that 4-AP exerts a significant inhibitory effect on A549/CDDP cell growth.

4-AP induces cell apoptosis of A549/CDDP cells. A549/CDDP cells revealed typical features of apoptosis including nuclear chromatin condensation and the appearance of apoptotic body with nuclear membrane observed by electron microscopy after treated with $7 \mathrm{mM}$ 4-AP for $48 \mathrm{~h}$ (Fig. 2A). Annexin $\mathrm{V}$-APC/7-ADD was employed to explore obvious enhanced cell apoptosis of A549/CDDP induced by 4-AP (Fig. 2B).

4-AP enhances the sensitivity of A549/CDDP cells to DDP. The IC50 of DDP for A549/CDDP cells was $75 \pm 2.36 \mu \mathrm{M}$, while the IC50 values decreased to $62.5 \pm 1.86,32.14 \pm 1.92$ and $0.00 \pm 0.03 \mu \mathrm{M}$ respectively in the presence of 3,6 and $10 \mathrm{mM}$ 4-AP (Fig. 3). 4-AP significantly enhanced A549/CDDP cell chemosensitivity to DDP.

4-AP upregulates the expression of PTEN and modulates PI3K/Akt signal and its downstream cell cycle and apoptosis-related proteins in A549/CDDP cells. We examined that 4-AP not only significantly increased the expression of PTEN, but also suppressed the expression of pPI3K (Tyr458) and pAkt (Ser473) (Fig. 4A). However, no alterations in PI3K and Akt expression were observed (Fig. 4A).

Cell cycle and apoptosis has been reported as downstream signal of PI3K/Akt pathway (9). 4-AP treatment suppressed the expression of CCND1 and CDK4, and elevated the expression of tumor suppressor p21 (Fig. 4B). Moreover, the expression

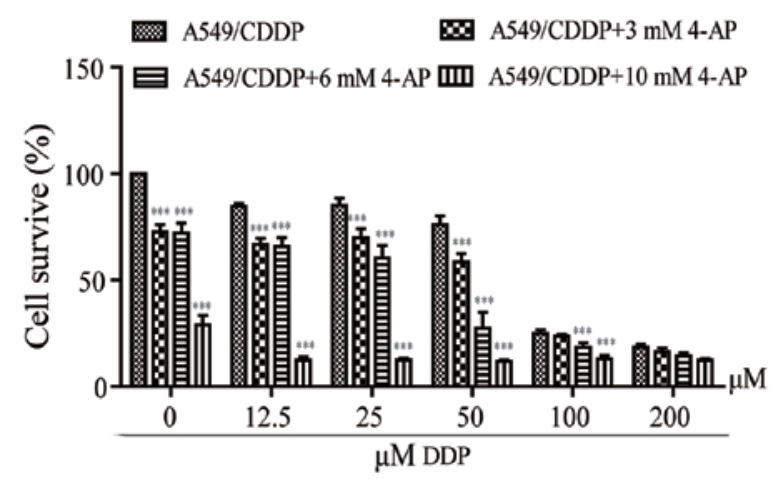

Figure 3. 4-AP enhances the sensitivity of A549/CDDP cells to DDP. A549/CDDP cells were treated with different concentrations of 4-AP (3, 6 and $10 \mathrm{mM})$ and $\mathrm{DDP}(0,12.5,25,50,100$ and $200 \mu \mathrm{M})$ for $48 \mathrm{~h}$. 4-AP sensitized A549/CDDP cells to DDP. Data were presented as mean \pm standard deviation for three independent experiments $\left({ }^{* * * *} \mathrm{P}<0.001\right.$ vs. the $0 \mu \mathrm{M}$ A549/CDDP group). 4-AP, 4-aminopyridine; DDP, cisplatin.

of apoptosis-related proteins including $\mathrm{Bcl} 2$, pro-caspase 9 and pro-caspase 3 was inhibited after 4-AP treatment, while the expression of cleaved caspase 9 and cleaved caspase 3 was induced (Fig. 4B).

PTEN knockdown partially increased aggressive phenotypes via upregulating PI3K/Akt signal in 4-AP-treated A549/CDDP cells. To better understand the role of PTEN in 4-AP-treated A549/CDDP cells, siRNA transfection was employed to knockdown PTEN expression. Knockdown efficiency was evaluated by western blot (Fig. 5A). Transiently transfecting PTEN siRNA into 4-AP-treated A549/CDDP cells not only significantly enhanced cell growth (Fig. 5B), but also inhibited cell apoptosis in A549/CDDP cells (Fig. 5C). Further, PTEN knockdown upregulated the expression of pPI3K (Tyr458), pAkt (Ser473), cyclin D1 and Bcl2 (Fig. 5D). These results indicated that PTEN knockdown could partially increase the aggressive phenotypes via upregulating PI3K/Akt signal in A549/CDDP cells with 4-AP treatment.

Taken together, our results demonstrated that 4-AP inhibited cell growth, induced cell apoptosis and sensitized A549/CDDP cells to DDP through upregulating PTEN. 
A
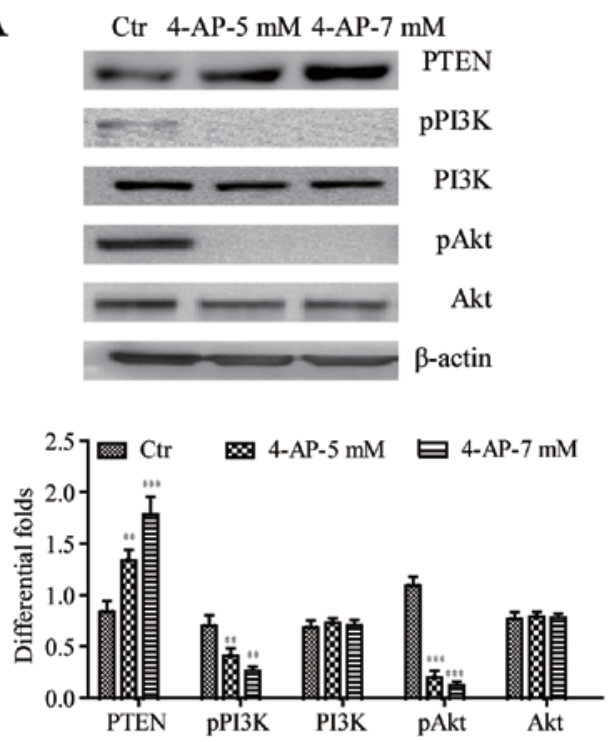

B

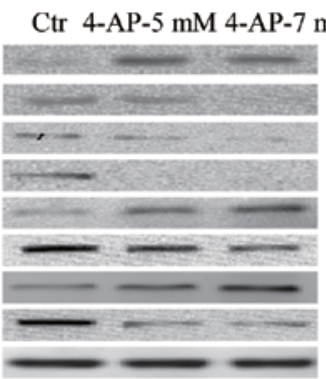

p21

CDK4

CCND1

$\mathrm{Bcl} 2$

cleaved-caspase 9

pro-caspase 9

cleaved-caspase 3

pro-caspase 3

$\beta$-actin

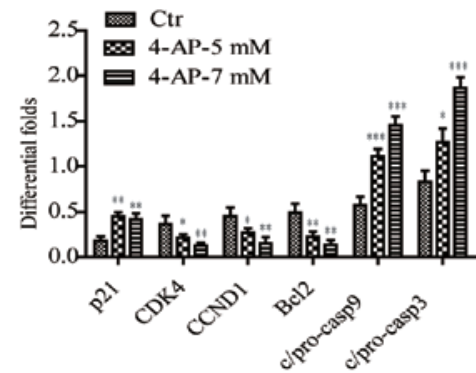

Figure 4. 4-AP upregulates the expression of PTEN and modulates PI3K/Akt signal and its downstream cell cycle and apoptosis-related proteins in A549/CDDP cells. (A) 4-AP suppressed the expression of pPI3K (Tyr458) and pAkt (Ser473), as well as elevated the expression of PTEN. No alterations in PI3K and Akt expression were observed. (B) 4-AP suppressed the expression of CDK4, CCND1, Bc12, pro-caspase 9 and pro-caspase 3, and elevated the expression of tumor suppressor $\mathrm{p} 21$, cleaved caspase 9 and cleaved caspase 3 . $\beta$-actin served as the internal control ${ }^{*} \mathrm{P}<0.05,{ }^{* * *} \mathrm{P}<0.01$ and ${ }^{* * * *} \mathrm{P}<0.001$ vs. the control. 4-AP, 4 -aminopyridine; PTEN, phosphatase and tensin homolog; PI3K, phosphoinositide 3-kinase; p, phosphorylated; Bcl2, B-cell lymphoma 2; CDK4, cyclin-dependent kinase 4; CCND1, cyclin-D1; Ctr, control.

A


B

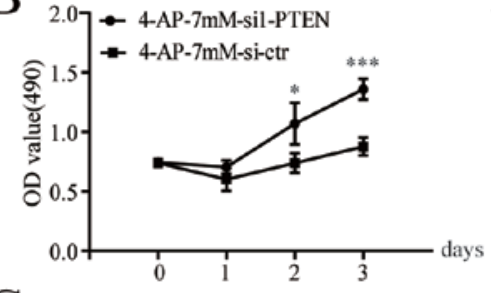

$\mathrm{D}$

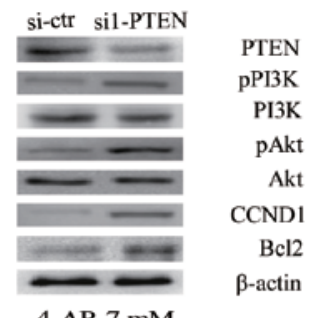

$\mathrm{C}$

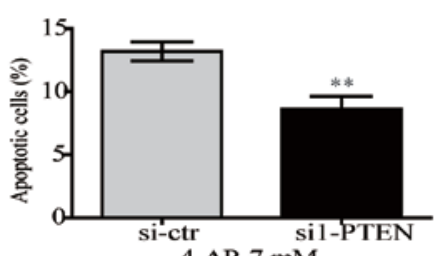

4-AP-7 mM

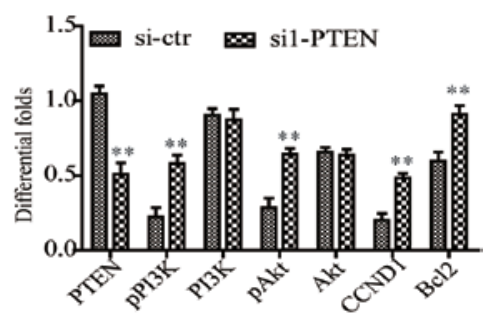

Figure 5. PTEN knockdown partially increased aggressive phenotypes via upregulating the PI3K/Akt signaling pathway in 4-AP-treated A549/CDDP cells. (A) Decreased PTEN protein levels were detected in A549/CDDP cells transfected with PTEN siRNA by western blot analysis. ${ }^{* *} \mathrm{P}<0.01$ and ${ }^{* * *} \mathrm{P}<0.001$ vs. the si-ctr group. (B) PTEN knockdown enhanced cell viability in 4-AP-treated A549/CDDP cells ( ${ }^{*}<0.05,{ }^{* * *} \mathrm{P}<0.001$ vs. the $4-\mathrm{AP}-7 \mathrm{mM}-\mathrm{si}-\mathrm{ctr}$ group). (C) Transiently downregulated PTEN dramatically inhibited cell apoptosis of 4-AP-treated A549/CDDP cells (**P<0.01). (D). PTEN knockdown upregulated the expression of pPI3K (Tyr458), pAkt (Ser473), CCND1 and Bcl2 in A549/CDDP cells. No alterations in PI3K and Akt expression were observed. $\beta$-actin served as the internal control ( ${ }^{* *} \mathrm{P}<0.01$ vs. the si-ctr group). 4-AP, 4-aminopyridine; PTEN, phosphatase and tensin homolog; ctr, control; Akt, protein kinase B; PI3K, phosphoinositide 3-kinase; p, phosphorylated.

\section{Discussion}

4-AP, one of the most commonly used $\mathrm{K}^{+}$channel inhibitors, suppresses proliferation and induces apoptosis in various types of cancer cells (4-7). However, the effect of 4-AP in A549/CDDP is largely unknown yet. In the present investigation, 4-AP played an important role in proliferation and apoptosis of A549/CDDP cells, which is consistent with previous results (4-7). In addition, 4-AP enhances the sensitivity of A549/CDDP cells to DDP. These findings suggest that 4-AP may have a wide range of antitumor effects.

In this study, we detected that 4-AP inhibited cell growth of A549/CDDP in vitro and in vivo, and retarded cell cycle progression. It is well known that high proliferative activity of tumor cells is associated with the increased cell-cycle transition (12). CCND1, a classic oncogenic protein of cell cycle signal, promotes cell proliferation and the beginning of $\mathrm{S}$ phase in the cell cycle in many cancers $(13,14)$. Here, we found that 4-AP-mediated 
growth suppression attributed to cell cycle transition obstacle by repressing the expression of cell cycle G1/S checkpoint proteins CCND1 and CDK4, and inducing the expression of p21.

Apoptosis, also called type 1 cell death, may be defined as suicidal cell death with a particular morphology involving nuclear chromatin condensation $(15,16)$. As we know the aim of anti-cancer therapy is to induce apoptosis of tumor cells. In this study, we found that 4-AP induced cell apoptosis of A549/CDDP. 4-AP-mediated cell apoptosis attributed to repressing the expression of $\mathrm{Bcl} 2$, an oncogenic protein inhibiting programmed cell death (17), and suppressing the expression of pro-caspase 9 and pro-caspase 3, which typically requires processing at caspase cleavage sites to generate the active enzyme cleaved caspase 9 and 3 (18) Once an initiator caspase is activated, it processes others that cleave a host of cellular proteins. A serial cascade reaction of caspase activation sentences cell to death (17).

PI3K/Akt, a classical signal pathway, inhibits cell apoptosis and induces cell-cycle progression (9). In this study, we found that 4-AP treatment significantly suppressed the expression of pPI3K and pAkt, and its downstream cell cycle and apoptosis signals. Further, PTEN, a well-known tumor suppressor that inhibits the activation of PI3K/Akt $(19,20)$, was found to be upregulated in 4-AP treated cells. Knocking down PTEN expression could increase the aggressive phenotypes and activate PI3K/Akt signal in A549/CDDP cells with 4-AP treatment. These findings suggest that 4-AP-mediated promotion of PTEN downregulates PI3K/Akt signaling, which in turn inhibits cell growth and induces cell apoptosis in A549/CDDP cells.

DDP is the most commonly used anti-cancer drug (21). However, chemotherapy resistance usually occurs mainly due to DDP resistance, the tolerance of cancer cells to DDP-induced apoptosis in lung cancers (3). The present study found 4-AP sensitized A549/CDDP cells to DDP in the presence of 4-AP and DDP together. These indicate that 4-AP may be used to improve the efficacy of DDP-based chemotherapy in patients with lung cancer.

Taken together, our results demonstrated that 4-AP inhibited cell growth, induced cell apoptosis and enhanced the sensitivity of A549/CDDP cells to DDP through upregulating PTEN. 4-AP may be used as an adjuvant therapy to improve the efficacy of patients with DDP-resistant lung cancers.

\section{Acknowledgements}

The present study was supported by National Nature Science Fund of China (grant nos. 81401906, 81502532) (http://www.nsfc.gov.cn), Natural Science Foundation of Guangdong Province (2014A030310239) and Doctoral Fund of Affiliated Hospital of Guangdong Medical University (grant no. BJ20150003).

\section{References}

1. Califano R, Karamouzis MV, Banerjee S, de Azambuja E, Guarneri V, Hutka M, Jordan K, Kamposioras K, Martinelli E, Corral J, et al: Use of adjuvant chemotherapy (CT) and radiotherapy (RT) in incompletely resected (R1) early stage non-small cell lung cancer (NSCLC): A European survey conducted by the European society for medical oncology (ESMO) young oncologists committee. Lung Cancer 85: 74-80, 2014.
2. Siegel R, Ma J, Zou Z and Jemal A: Cancer statistics, 2014. CA Cancer J Clin 64: 9-29, 2014.

3. Stewart DJ: Mechanisms of resistance to cisplatin and carboplatin. Crit Rev Oncol Hematol 63: 12-31, 2007.

4. Chin LS, Park CC, Zitnay KM, Sinha M, DiPatri AJ Jr, Perillán P and Simard JM: 4-Aminopyridine causes apoptosis and blocks an outward rectifier $\mathrm{K}+$ channel in malignant astrocytoma cell lines. J Neurosci Res 48: 122-127, 1997.

5. Kim JA, Kang YS, Jung MW, Kang GH, Lee SH and Lee YS: $\mathrm{Ca} 2+$ influx mediates apoptosis induced by 4 -aminopyridine, a $\mathrm{K}+$ channel blocker, in HepG2 human hepatoblastoma cells. Pharmacology 60: 74-81, 2000.

6. Wang W, Xiao J, Adachi M, Liu Z and Zhou J: 4-aminopyridine induces apoptosis of human acute myeloid leukemia cells via increasing [Ca2+]i through P2X7 receptor pathway. Cell Physiol Biochem 28: 199-208, 2011.

7. Huang L, Li B, Li W, Guo H and Zou F: ATP-sensitive potassium channels control glioma cells proliferation by regulating ERK activity. Carcinogenesis 30: 737-744, 2009.

8. Dai T, Zhou Q, Zeng X, He J, Yang Y, Li C, Ren D, Liu L and Liao B: A study on the characteristics of the membrane potassium channels in human non-small cell lung cancer cell. Zhongguo Fei Ai Za Zhi 4: 281-286, 2001 (In Chinese).

9. Zhen Y, Liu Z, Yang H, Yu X, Wu Q, Hua S, Long X, Jiang Q, Song Y, Cheng C, et al: Tumor suppressor PDCD4 modulates miR-184-mediated direct suppression of C-MYC and BCL2 blocking cell growth and survival in nasopharyngeal carcinoma. Cell Death Dis 4: e872, 2013.

10. Ru Q, Tian X, Wu YX, Wu RH, Pi MS and Li CY: Voltage-gated and ATP-sensitive $\mathrm{K}+$ channels are associated with cell proliferation and tumorigenesis of human glioma. Oncol Rep 31: 842-848, 2014.

11. Wang H, Wu Q, Liu Z, Luo X, Fan Y, Liu Y, Zhang Y, Hua S, Fu Q, Zhao M, et al: Downregulation of FAP suppresses cell proliferation and metastasis through PTEN/PI3K/AKT and Ras-ERK signaling in oral squamous cell carcinoma. Cell Death Dis 5: e1155, 2014.

12. Huang L, Wang HY, Li JD, Wang JH, Zhou Y, Luo RZ, Yun JP, Zhang Y, Jia WH and Zheng M: KPNA2 promotes cell proliferation and tumorigenicity in epithelial ovarian carcinoma through upregulation of c-Myc and downregulation of FOXO3a. Cell Death Dis 4: e745, 2013.

13. Jiang W, Kahn SM, Zhou P, Zhang YJ, Cacace AM, Infante AS, Doi S, Santella RM and Weinstein IB: Overexpression of cyclin D1 in rat fibroblasts causes abnormalities in growth control, cell cycle progression and gene expression. Oncogene 8: 3447-3457, 1993.

14. Baldin V, Lukas J, Marcote MJ, Pagano M and Draetta G: Cyclin D1 is a nuclear protein required for cell cycle progression in G1. Genes Dev 7: 812-821, 1993.

15. Kroemer G, El-Deiry WS, Golstein P, Peter ME, Vaux D, Vandenabeele P, Zhivotovsky B, Blagosklonny MV, Malorni W, Knight RA, et al: Classification of cell death: Recommendations of the nomenclature committee on cell death. Cell Death Differ 12 (Suppl 2): S1463-S1467, 2005.

16. Galluzzi L, Maiuri MC, Vitale I, Zischka H, Castedo M, Zitvogel L and Kroemer G: Cell death modalities: Classification and pathophysiological implications. Cell Death Differ 14: 1237-1243, 2007.

17. Cory S and Adams JM: The Bcl2 family: Regulators of the cellular life-or-death switch. Nat Rev Cancer 2: 647-656, 2002.

18. Shi Y: Mechanisms of caspase activation and inhibition during apoptosis. Mol Cell 9: 459-470, 2002.

19. Dong-Dong L, Xi-Ran Z and Xiang-Rong C: Expression and significance of new tumor suppressor gene PTEN in primary liver cancer. J Cell Mol Med 7: 67-71, 2003.

20. Luo H, Yang Y, Duan J, Wu P, Jiang Q and Xu C: PTEN-regulated $\mathrm{AKT} / \mathrm{FoxO} 3 \mathrm{a} / \mathrm{Bim}$ signaling contributes to reactive oxygen species-mediated apoptosis in selenite-treated colorectal cancer cells. Cell Death Dis 4: e481, 2013.

21. Florea AM and Büsselberg D: Cisplatin as an anti-tumor drug: Cellular mechanisms of activity, drug resistance and induced side effects. Cancers (Basel) 3: 1351-1371, 2011. 